Eur. J. Math. Appl. (2021)1:2

URL: http://ejma.euap.org

(C) 2021 European Journal of Mathematics and Applications

\title{
NONLOCAL ITERATIVE DIFFERENTIAL EQUATIONS UNDER GENERALIZED FRACTIONAL DERIVATIVES
}

\author{
D. VIVEK ${ }^{1}$, E. M. ELSAYED ${ }^{2 *}$ AND K. KANAGARAJAN ${ }^{3}$
}

\begin{abstract}
The objective of this paper is to study the existence and uniqueness of solutions of iterative differential equations with nonlocal conditions under $\psi$-type fractional derivative (or generalized fractional derivative).
\end{abstract}

\section{INTRODUCTION}

The nonlocal condition can be applied in physics with better effect than the classical initial value problem. Nonlocal conditions were initiated by Byszewski [4] when he proved the existence and uniqueness of mild and classical solutions of nonlocal Cauchy problems. As remarked by Byszewski [5] and Deng [7], the nonlocal condition can be more useful thatn standard initial condition to describe some physical phenomena.

Many recent papers have dealt with the existence, uniqueness and other properties of solutions of special forms of the iterative fractional differential equations, for example [3, 9, 10]. Recently, Kharat and Jagtap [11] have investigated the existence and uniquness of solutions of special form of iterative fractioanl differential equations.

Motivated by [11], in this paper, we consider the class of $\psi$-type fractional iterative differential equation of the type

$$
\begin{aligned}
& \mathscr{D}^{\alpha ; \psi} x(t)=f(t, x(x(t))), \quad 0<\alpha<1, \\
& x(0)+g(x)=x_{0},
\end{aligned}
$$

where $\mathscr{D}^{\alpha ; \psi}$ is the $\psi$-type Riemann-Liouville fractional derivative of order $\alpha, f: J \times J \rightarrow J$, $g: C(J, J) \rightarrow J$ are continuous functions and $t, x_{0} \in J=[0, T]$.

The aim of the present paper is to prove the existence and uniqueness of solutions of iterative differential equations under $\psi$-type fractional derivative. The main tools used in our analysis are based on the theory of fractional calculus and fixed point theorems.

\footnotetext{
${ }^{1}$ Department of Mathematics, PSG College of Arts \& Science, Coimbatore-641014, India

${ }^{2}$ Department of Mathematics, Faculty of Science,King Abdulaziz University, Jeddah 21589 , Saudi Arabia

${ }^{3}$ Department of Mathematics, Sri Ramakrishna Mission Vidyalaya College of Arts and Science, Coimbatore-641020, India

${ }^{*}$ Corresponding AUthor

E-mail addresses: peppyvivek@gmail.com, emmelsayed@yahoo.com, kanagarajank@gmail.com.

Key words and phrases. $\psi$-fractional derivative; nonlocal initial value problem; iterative differential equations; existence; fixed point.
}

Received 08/08/2021. 


\section{Prerequisites}

First we state some definitions, results and hypotheses which are useful for our analysis.

Definition 2.1. [1] The $\psi$-type fractional order integral of the function $f$ of order $\gamma>0$ is defined by

$$
\mathscr{I}_{a}^{\gamma ; \psi} f(t)=\frac{1}{\Gamma(\gamma)} \int_{a}^{t} \psi^{\prime}(s)(\psi(t)-\psi(s))^{\gamma-1} f(s) d s
$$

where $\Gamma$ is gamma function.

Definition 2.2. [1] The $\psi$-type fractional order derivative of the function $f$ of order $0 \leq \gamma<1$ is defined by

$$
\mathscr{D}_{a}^{\gamma ; \psi}=\frac{1}{\Gamma(1-\gamma)}\left(\frac{d}{d t}\right) \int_{a}^{t} \psi^{\prime}(s)(\psi(t)-\psi(s))^{-\gamma} d s=\frac{d}{d t} \mathscr{I}_{a}^{1-\gamma ; \psi} f(t) .
$$

Let $(X, d)$ be a metric space. A mapping $P: X \rightarrow X$ is said to be $\nu$-contraction if there exists $\nu \in[0,1)$ such that

$$
d(P x, P y) \leq \nu d(x, y), \quad \forall x, y \in X .
$$

If $\nu=1$, then the mapping $P$ is said to be non-expansive.

Theorem 2.3. [2] Let $K$ be a nonempty closed convex and bounded subset of a uniformly Banach space $E$. Then any non-expansive mapping $P: K \rightarrow K$ has at least a fixed point.

Definition 2.4. [11] Let $K$ be a convex subset of a normal linear space $E$ and let $P: K \rightarrow K$ be a self-mapping. Given an $x_{0} \in K$ and a real number $\lambda \in[0,1]$, the sequence $x_{n}$ defined by the formula

$$
x_{n+1}=(1-\lambda) x_{n}+\lambda P x_{n}, \quad n=0,1,2 \ldots
$$

is usually called Krasnoselskij iteration or Krasnoselskij-Mann iteration.

Definition 2.5. [11] Let $K$ be a convex subset of a normed linear space $E$ and let $P: E \rightarrow E$ be a self-mapping. Given an $x_{0} \in K$ and a real number $\lambda_{m} \in[0,1]$, the sequence $x_{n}$ defined by the formula

$$
x_{n+1}=\left(1-\lambda_{n}\right) x_{n}+\lambda_{n} P x_{n}, \quad n=0,1,2, \ldots
$$

is usually called Mann iteration.

Edelstein [8] proved that strict convexity of $E$ suffices for the Krasnoselskij iteration converges to a fixed point of $P$.

Lemma 2.6. [6] Let $K$ be a convex and compact subset of Banach space $E$ and let $P: K \rightarrow K$ be a non-expansive mapping. If the Mann iteration process $x_{n}$ satisfies the assumptions

(a) $x_{n} \in K$ for all positive integers $n$,

(b) $0 \leq \lambda_{n} \leq b<1$ for all positive integer $n$,

(c) $\sum_{n=0}^{\infty} \lambda_{n}=\infty$.

Then $x_{n}$ converges strongly to a fixed point of $P$. 
Lemma 2.7. [6] Let $K$ be a closed bounded convex subset of a real normed space $E$ and $P: K \rightarrow K$ be a non-expansive mapping. If $I-P$ maps closed bounded subset of $E$ into closed subset of $E$ and $x_{n}$ is the Mann iteration with $\lambda_{n}$ satisfying assumptions (a)-(c) in Lemma 2.6, then $x_{n}$ converges strongly to a fixed point of $P$ in $K$.

Let $C(J, J)$ be the Banach space of all continuous functions from $J$ into $J$ endowed with the norm $\|x\|=\sup \{|x(t)|: t \in J\}, M_{t}=\max \{t, T-t\}$ and

$$
C_{L, \alpha ; \psi}=\left\{x:\left|x\left(t_{1}\right)-x\left(t_{2}\right)\right| \leq \frac{L}{\Gamma(\alpha+1)}\left|\left(\psi\left(t_{1}\right)\right)-\left(\psi\left(t_{2}\right)\right)\right|^{\alpha}, \quad \forall t_{1}, t_{2} \in J, L>0\right\} .
$$

It is clear that $C_{t, \alpha ; \psi}$ is a nonempty convex and compact subset of the Banach space $(C[J],\|\cdot\|)$. For the convenience, we list the following hypotheses used in our further discussion.

(H1) For all $t, x_{1}, x_{2} \in J$ there exists a constant $l>0$ such that

$$
\left|f\left(t, x_{1}\right)-f\left(t, x_{2}\right)\right| \leq l\left|x_{1}-x_{2}\right|
$$

and a constant $l_{g}>0$ such that

$$
\left\|g\left(x_{1}\right)-g\left(x_{2}\right)\right\| \leq l_{g}\left\|x_{1}-x_{2}\right\| .
$$

Also for arbitary $x \in C(J, J)$, there exists a constant $N>0$ such that

$$
\|g(x)\| \leq N .
$$

(H2) If there exist a constant $L$ such that $\left|x\left(t_{1}\right)-x\left(t_{2}\right)\right| \leq \frac{L}{\Gamma(\alpha+1)}\left|\left(\psi\left(t_{1}\right)\right)-\left(\psi\left(t_{2}\right)\right)\right|^{\alpha}$ then

$$
M=\max \{|f(t, x)|:(t, x) \in J \times J\} \leq \frac{L}{2} .
$$

(H3) One of the following conditions holds:

(a) $M \frac{(\psi(T))^{\alpha}}{\Gamma(\alpha+1)} \leq M_{x_{0}}$, where $M_{x_{0}}=\max \left\{x_{0}+N, T-\left(x_{0}+N\right)\right\}$;

(b) $x_{0}-N=0, M \frac{(\psi(T))^{\alpha}}{\Gamma(\alpha+1)} \leq T-\left(x_{0}+N\right), f(t, x \geq 0)$ for all $t, x \in J$;

(c) $x_{0}-N=T, M \frac{(\psi(T))^{\alpha}}{\Gamma(\alpha+1)} \leq x_{0}-N, f(t, x) \geq 0$ for all $t, x \in J$,

(H4) $x_{0}+N \leq \frac{\delta\left(\psi\left(t_{0}\right)\right)^{\alpha}}{2 \Gamma(\alpha+1)}, 0 \neq t_{0} \in J, \delta \in(0,1)$.

(H5) If there exist a constant $L$ such that $\left|x\left(t_{1}\right)-x\left(t_{2}\right)\right| \leq \frac{L}{\Gamma(\alpha+1)}\left|\left(\psi\left(t_{1}\right)\right)-\left(\psi\left(t_{2}\right)\right)\right|^{\alpha}$ then $M=\min \left\{\frac{\delta}{2}, \frac{L}{2}\right\}$.

\section{MAin Results}

In this section,we state and prove results related to existence and uniqeness of solutions of iterative differential equations under $\psi$-type fractional derivative.

Theorem 3.1. Suppose that hypotheses (H1)-(H3) are satisfied and

$$
\left\{l_{g}+\frac{(\psi(T))^{\alpha} l}{\Gamma(\alpha+1)}\left[\frac{L}{\Gamma(\alpha+1)}+1\right]\right\} \leq 1
$$

then there exists at least one solution of the system (1)-(2) in $C_{L, \alpha ; \psi}$ which can be approximated by the Krasnoselskij iteration

$$
x_{n+1}=(1-\lambda) x_{n}+\lambda x_{0}-\lambda g(x)+\lambda \frac{1}{\Gamma(\alpha)} \int_{0}^{t} \psi^{\prime}(s)((\psi(t))-(\psi(s)))^{\alpha-1} f\left(\tau, x_{n}\left(x_{n}(t)\right)\right) d \tau,
$$

where $\lambda \in(0,1)$ and $x_{1} \in C_{L, \alpha ; \psi}$ is arbitrary. 
Proof. Consider the integral operator $P: C_{L, \alpha ; \psi} \rightarrow C(J)$ defined by

$$
P x(t)=x_{0}+g(x)+\frac{1}{\Gamma(\alpha)} \int_{0}^{t} \psi^{\prime}(s)((\psi(t))-(\psi(s)))^{\alpha-1} f\left(\tau, x_{n}\left(x_{n}(t)\right)\right) d \tau, \quad t \in J
$$

Any fixed point of the equation $x=P x$ is a solution of initial value problem (1)-(2).

First we show that $C_{L, \alpha ; \psi}$ is invariant set with respect to $P$, i.e., $P\left(C_{L, \alpha ; \psi}\right) \subset C_{L, \alpha ; \psi}$. Making use of hypothesis (H3)(a), we have

$$
\begin{aligned}
|P x(t)| & \leq x_{0}+|g(x)|+\left|\frac{1}{\Gamma(\alpha)} \int_{0}^{t} \psi^{\prime}(s)((\psi(t))-(\psi(s)))^{\alpha-1} f\left(\tau, x_{n}\left(x_{n}(t)\right)\right) d \tau\right| \\
& \leq x_{0}+|g(x)|+M \frac{(\psi(T))^{\alpha}}{\Gamma(\alpha+1)} \\
& \leq x_{0}+|g(x)|+M_{x_{0}} \\
& \leq(\psi(T))
\end{aligned}
$$

and

$$
\begin{aligned}
|P x(t)| & \geq x_{0}+|g(x)|-\left|\frac{1}{\Gamma(\alpha)} \int_{0}^{t} \psi^{\prime}(s)((\psi(t))-(\psi(s)))^{\alpha-1} f\left(\tau, x_{n}\left(x_{n}(t)\right)\right) d \tau\right| \\
& \leq x_{0}+|g(x)|-M \frac{(\psi(T))^{\alpha}}{\Gamma(\alpha+1)} \\
& \leq x_{0}+|g(x)|-M_{x_{0}} \geq 0 .
\end{aligned}
$$

Thus $P x(t) \in J, t \in J$.

Similarly, we get the result using hypotheses (H3)(b) and (H3)(c).

Using hypotheses (H2), for every $t_{1}, t_{2} \in J$, we obtain

$$
\begin{aligned}
& \left|(P x)\left(t_{1}\right)-(P x)\left(t_{2}\right)\right| \\
& =\mid \frac{1}{\Gamma(\alpha)} \int_{0}^{t_{1}} \psi^{\prime}(s)\left(\left(\psi\left(t_{1}\right)\right)-(\psi(\tau))\right)^{\alpha-1} f\left(\tau, x_{n}\left(x_{n}(t)\right)\right) d \tau \\
& -\frac{1}{\Gamma(\alpha)} \int_{0}^{t_{2}} \psi^{\prime}(s)\left(\left(\psi\left(t_{2}\right)\right)-(\psi(\tau))\right)^{\alpha-1} f\left(\tau, x_{n}\left(x_{n}(t)\right)\right) d \tau \mid \\
& \leq 2 M \frac{\left|\left(\psi\left(t_{1}\right)\right)-\left(\psi\left(t_{2}\right)\right)\right|^{\alpha}}{\Gamma(\alpha+1)} \\
& \leq L \frac{\left|\left(\psi\left(t_{1}\right)\right)-\left(\psi\left(t_{2}\right)\right)\right|^{\alpha}}{\Gamma(\alpha+1)} .
\end{aligned}
$$

Hence $P x \in C_{L, \alpha ; \psi}$ whenever $x \in C_{L, \alpha ; \psi}$. Therefore, $P: C_{L, \alpha ; \psi} \rightarrow C_{L, \alpha ; \psi}$, i.e., $P$ is a self mapping. 
Using hypotheses (H1) for $x, y \in C_{L, \alpha ; \psi}$ and $t \in J$, we have

$$
\begin{aligned}
& |(P x)(t)-(P y)(t)| \\
& \leq\|g(x)-g(y)\| \\
& +\mid \frac{1}{\Gamma(\alpha)} \int_{0}^{t} \psi^{\prime}(s)((\psi(t))-(\psi(\tau)))^{\alpha-1} f\left(\tau, x_{n}\left(x_{n}(t)\right)\right) d \tau \\
& -\frac{1}{\Gamma(\alpha)} \int_{0}^{t} \psi^{\prime}(s)((\psi(t))-(\psi(\tau)))^{\alpha-1} f\left(\tau, y_{n}\left(y_{n}(t)\right)\right) d \tau \mid \\
& \leq l_{g}\|x-y\|+\frac{1}{\Gamma(\alpha)} \int_{0}^{t} \psi^{\prime}(s)((\psi(t))-(\psi(\tau)))^{\alpha-1}\left|f\left(\tau, x_{n}\left(x_{n}(t)\right)\right)-f\left(\tau, y_{n}\left(y_{n}(t)\right)\right)\right| d \tau \\
& \leq l_{g}\|x-y\|+\frac{l}{\Gamma(\alpha)} \int_{0}^{t} \psi^{\prime}(s)((\psi(t))-(\psi(\tau)))^{\alpha-1}|x(x(\tau))-y(y(\tau))| d \tau \\
& \leq l_{g}\|x-y\| \\
& +\frac{l}{\Gamma(\alpha)} \int_{0}^{t} \psi^{\prime}(s)((\psi(t))-(\psi(\tau)))^{\alpha-1}[|x(x(\tau))-x(y(\tau))|+|x(y(\tau))-y(y(\tau))|] d \tau \\
& \leq\left\{l_{g}+\frac{(\psi(T))^{\alpha} l}{\Gamma(\alpha+1)}\left[\frac{L}{\Gamma(\alpha+1)}+1\right]\right\}\|x-y\| .
\end{aligned}
$$

Now, by taking supremum we get,

$$
\|(P x)-(P y)\| \leq\left\{l_{g}+\frac{(\psi(T))^{\alpha} l}{\Gamma(\alpha+1)}\left[\frac{L}{\Gamma(\alpha+1)}+1\right]\right\}\|x-y\| .
$$

In view of equation (5)

(i) if $\left\{l_{g}+\frac{(\psi(T))^{\alpha} l}{\Gamma(\alpha+1)}\left[\frac{L}{\Gamma(\alpha+1)}+1\right]\right\}<1$, then $P$ is a contraction mapping and hence by Banach fixed point theorem equation (1)-(2) has a unique solution.

(ii) if $\left\{l_{g}+\frac{(\psi(T))^{\alpha} l}{\Gamma(\alpha+1)}\left[\frac{L}{\Gamma(\alpha+1)}+1\right]\right\}=1$, then $P$ is nonexpansive and hence it is continuous.

Thus Theorem 2.3 implies that equation (1)-(2) has a solution in $C_{L, \alpha ; \psi}$.

Finally, by applying Lemma 2.6 and Lemma 2.7, we obtain the second part of the theorem.

Next we estabilish the solution of equation (1)-(2) in a subset of $C_{L, \alpha ; \psi}$ defined by

$$
C_{L, \alpha ; \psi, \delta}=\left\{x \in C_{L, \alpha ; \psi}: x(t) \leq \frac{\delta(\psi(t))^{\alpha}}{\Gamma(\alpha+1)}, \quad \text { for all } \quad t \in J\right\}, \quad \delta \in(0,1) .
$$

It is clear that $C_{L, \alpha ; \psi, \delta}$ is non-empty, convex and compact subset in $C(J)$.

Theorem 3.2. Assume that hypotheses (H1), (H3)-(H5) are satisfied. If

$$
\begin{aligned}
L_{1} & =\left\{l_{g}+\frac{(\psi(T))^{\alpha-1} l}{q \Gamma(\alpha)}\left(\frac{L}{\Gamma(\alpha+1)}\left|\left(1-\exp \left(-q\left(\psi(t)-\psi\left(t_{0}\right)\right)\right)\right)\right|\right.\right. \\
& \left.\left.+\frac{1}{\delta}\left|\exp (q(\delta-1)(\psi(t)))-\exp \left(q\left(\delta \psi\left(t_{0}\right)-\psi(t)\right)\right)\right|\right)\right\} \\
& \leq l
\end{aligned}
$$

then there exists at least one solution of the system (1)-(2) in $C_{L, \alpha ; \psi, \delta}$ which can be approximated by the Krasnoselskij iteration

$$
x_{n+1}=(1-\lambda) x_{n}+\lambda x_{0}-\lambda g(x)+\lambda \frac{1}{\Gamma(\alpha)} \int_{0}^{t} \psi^{\prime}(s)((\psi(t))-(\psi(\tau)))^{\alpha-1} f\left(\tau, x_{n}\left(x_{n}(t)\right)\right) d \tau,
$$


where $\lambda \in(0,1)$ and $x_{1} \in C_{L, \alpha ; \psi, \delta}$ is arbitrary.

Proof. Let $C(J)$ be the Banach space endowed with the Bieleckis norm given by the formula

$$
\|x\|_{B}=\max _{t \in J}\left\{\|x(t)\| \exp \left(-q\left(\psi(t)-\psi\left(t_{0}\right)\right)\right), \quad q>0, t>t_{0}\right\}
$$

Let $P$ be defined as in the proof of Theorem 3.1, by assumptions (H1), (H3)-(H5), it is sufficient to prove that, if $x \in C_{L, \alpha ; \psi, \delta}$ then $P x \in C_{L, \alpha ; \psi, \delta}$.

For $x \in C_{L, \alpha ; \psi, \delta}$ and $t \in J$, we have

$$
\begin{aligned}
P x(t) & \leq x_{0}+N+M \frac{(\psi(t))^{\alpha}}{\Gamma(\alpha+1)} \\
& \leq x_{0}+N+\frac{M\left((\psi(t))^{\alpha}-\left(\psi\left(t_{0}\right)\right)^{\alpha}\right)+M\left(\psi\left(t_{0}\right)\right)^{\alpha}}{\Gamma(\alpha+1)} \\
& \leq \frac{\delta(\psi(t))^{\alpha}}{2 \Gamma(\alpha+1)}, \quad t>t_{0} .
\end{aligned}
$$

This shows that $P x \in C_{L, \alpha ; \psi, \delta}$ and hence $C_{L, \alpha ; \psi, \delta}$ is invariant under $P$.

Now, for $x, y \in C_{L, \alpha ; \psi, \delta}$ and $t \in J$, we get

$$
\begin{aligned}
& |(P x)(t)-(P y)(t)| \\
& \leq\|g(x)-g(y)\| \\
& +\mid \frac{1}{\Gamma(\alpha)} \int_{0}^{t} \psi^{\prime}(s)((\psi(t))-(\psi(\tau)))^{\alpha-1} f\left(\tau, x_{n}\left(x_{n}(t)\right)\right) d \tau \\
& \frac{1}{\Gamma(\alpha)} \int_{0}^{t} \psi^{\prime}(s)((\psi(t))-(\psi(\tau)))^{\alpha-1} f\left(\tau, y_{n}\left(y_{n}(t)\right)\right) d \tau \mid \\
& \leq l_{g}\|x-y\| \\
& +\frac{(\psi(T))^{\alpha}}{\Gamma(\alpha)}\left|\int_{0}^{t}\left(\frac{L}{\Gamma(\alpha)}|x(\tau)-y(\tau)|+|x(y(\tau))-y(y(\tau))|\right) d \tau\right| \\
& \leq\left\{l_{g} \exp \left(q\left((\delta \psi(t))-\left(\psi\left(t_{0}\right)\right)\right)\right)+\frac{(\psi(T))^{\alpha-1} l}{\Gamma(\alpha)}\left(\left|\frac{L}{q \Gamma(\alpha+1)}\left(\exp \left(q\left(\psi(t)-\psi\left(t_{0}\right)\right)\right)-1\right)\right|\right.\right. \\
& \left.\left.+\frac{1}{q \delta}\left|\exp \left(q\left(\delta \psi(t)-\psi\left(t_{0}\right)\right)\right)-\exp \left(q\left(\delta \psi\left(t_{0}\right)-\psi\left(t_{0}\right)\right)\right)\right|\right)\right\}\|x-y\|_{B}
\end{aligned}
$$

Hence,

$$
\begin{aligned}
& |(P x)(t)-(P y)(t)| \exp \left(-q\left(\psi(t)-\psi\left(t_{0}\right)\right)\right) \\
& \leq\left\{l_{g}+\frac{(\psi(T))^{\alpha-1} l}{q \Gamma(\alpha)}\left(\frac{L}{\Gamma(\alpha+1)}\left|\left(1-\exp \left(-q\left(\psi(t)-\psi\left(t_{0}\right)\right)\right)\right)\right|\right.\right. \\
& \left.\left.+\frac{1}{\delta}\left|\exp (q(\delta-1)(\psi(t)))-\exp \left(q\left(\delta \psi\left(t_{0}\right)-\psi(t)\right)\right)\right|\right)\right\}\|x-y\|_{B} .
\end{aligned}
$$

Now, by taking maximum in the last inquality, we obtain

$$
\|P x-P y\|_{B} \leq L_{1}\|x-y\|_{B} .
$$

In view of equation (6)

(i) if $L_{1}<1$, then $P$ is a contraction mapping and hence by Banach fixed point theorem equation (1)-(2) has a unique solution in $C_{L, \alpha ; \psi, \delta}$. 
(ii) if $L_{1}=1$ then $P$ is nonexpansive and hence it is continuous. Thus Theorem 2.3 implies that equation (1)-(2) has a solution in $C_{L, \alpha ; \psi, \delta}$.

Finally, by applying Lemma 2.6 and Lemma 2.7, we obtain the second part of the theorem. This completes the proof.

\section{ACKNOWLEDGEMENT}

The authors are thankful to the anonymous reviewers and the handling editor for the fruitful comments that made the presentation of the work more interested.

\section{REFERENCES}

[1] R. Almeida, A Caputo fractional derivative of a function with respect to another function, Commun. Nonlinear Sci. Numer. Simul. 44 (2017) 460-481. https://doi.org/10.1016/j .cnsns.2016.09.006.

[2] V. Berinde, Iterative Approximation of Fixed Points, 2nd Ed., Springer Verlag, Berlin heidelberg, New York, 2007.

[3] V. Berinde, Existence and approximation of solutions of some first order iterative differential equations, Miskolc Math. Notes, 11 (2010) 13-26. https://doi.org/10.18514/MMn. 2010.256.

[4] L. Byszewski, Theorems about the existence and uniqueness of solutions of a semilinear evolution nonlocal Cauchy problem, J. Math. Anal. Appl. 162 (1991) 494-505. https://doi.org/10.1016/0022-247X (91) 90164-U.

[5] L. Byszewski, V. lakshmikantham, Theorem about the existence and uniqueness of solution of a nonlocal abstract Cauchy problem in a Banach space, Appl. Anal. 40 (1991), 11-19. https://doi.org/10.1080/ 00036819008839989.

[6] C. Chldume, Geometric Properties of Banach Spaces and Nonlinear Iterations, Springer Verlags, Berlin, Heidelberg New York, 2009.

[7] K. Deng, Exponential decay of solutions of semilinear parabolic equations with nonlocal initial conditions, J. Math. Anal. Appl. 179 (2) (1993) 630-637. https://doi.org/10.1006/jmaa.1993.1373.

[8] M. Edelstein, A remark on a theorem of M. A. Krasnoselski, Amer. Math. Monthly, 73 (1966), 509-510. https://doi.org/10.2307/2315474.

[9] R. Hilfer, Fractional Diffusion Based on Riemann-Liouville Fractional Derivatives, J. Phys. Chem. B. 104 (16) (2000) 3914-3917. https://doi.org/10.1021/jp9936289.

[10] R.W. Ibrahim, S. Momani, On the existence and uniqueness of solutions of a class of fractional differential equations, J. Math. Anal. Appl. 334 (2007) 1-10. https://doi.org/10.1016/j.jmaa.2006.12.036.

[11] V.V. Kharat, T.B. Jagtap, Existence of iterative fractional differential equation with nonlocal condition, J. Indian Math. Soc. 83 (1-2) (2016), 97-106. 